Check for updates

Cite this: Phys. Chem. Chem. Phys., 2021, 23, 5936

\title{
On the liquid demixing of water + elastin-like polypeptide mixtures: bimodal re-entrant phase behaviourtt
}

\author{
Tom Lindeboom, ${ }^{a}$ Binwu Zhao, ${ }^{\mathrm{b}}$ George Jackson, (D) ${ }^{\mathrm{a}}$ Carol K. Hall (D) ${ }^{\mathrm{b}}$ and \\ Amparo Galindo (D) *a
}

\begin{abstract}
Water + elastin-like polypeptides (ELPS) exhibit a transition temperature below which the chains transform from collapsed to expanded states, reminiscent of the cold denaturation of proteins. This conformational change coincides with liquid-liquid phase separation. A statistical-thermodynamics theory is used to model the fluid-phase behavior of ELPs in aqueous solution and to extrapolate the behavior at ambient conditions over a range of pressures. At low pressures, closed-loop liquid-liquid equilibrium phase behavior is found, which is consistent with that of other hydrogen-bonding solvent + polymer mixtures. At pressures evocative of deep-sea conditions, liquid-liquid immiscibility bounded by two lower critical solution temperatures (LCSTs) is predicted. As pressure is increased further, the system exhibits two separate regions of closed-loop of liquid-liquid equilibrium (LLE). The observation of bimodal LCSTs and two re-entrant LLE regions herald a new type of binary global phase diagram: Type XII. At highELP concentrations the predicted phase diagram resembles a protein pressure denaturation diagram; possible "molten-globule"-like states are observed at low concentration.
\end{abstract}

Received 22nd September 2020 Accepted 30th January 2021

DOI: $10.1039 / \mathrm{d} 0 \mathrm{cp} 05013 \mathrm{j}$

rsc.li/pccp been used as a proxy for elastin ${ }^{7,8}$ and for intrinsically disordered proteins (IDPs) in general. ${ }^{9}$ IDPs are responsible for key biological functions such as cell signalling, molecule recognition, and tissue elasticity. ${ }^{9}$ The disordered nature of IDPs is attributed to repetitive low-complexity amino acid sequences; ELP macromolecules consist of a repeating amino acid pentamer and can, therefore, be considered a minimal prototypical IDP. ${ }^{9}$

ELPs comprise repeat units of one of elastin's characteristic moieties: Valine-Proline-Glycine-Xaa-Glycine (VPGXG), where Xaa (X) is any amino acid apart from proline. ${ }^{10}$ Elastin is an extracellular matrix protein with unique viscoelastic properties, which are essential to the function of the connective tissue of vertebrates. $^{7,8,11}$ While most proteins denature upon heating, elastin has the unique behavior that it "folds" when the temperature is increased from below to above $\sim 40{ }^{\circ} \mathrm{C}:{ }^{7}$ this is known as inverse temperature transition behavior. ${ }^{7,10-14}$ The use of the term "folding" in this context reflects the fact that the polypeptide chain becomes more collapsed and ordered above the transition temperature $T_{\mathrm{t}} ;{ }^{7,11}$ the same phenomenon as observered for cold denaturation. In particular, the amino acid sequence VPGXG has been identified as the key to the characteristic thermoresponsive behavior of elastin. ${ }^{7}$ Like elastin, aqueous solutions of ELPs exhibit an inverse transition temperature, which is associated with the lower critical solution temperature (LCST) characterising the system. ${ }^{15-18}$ The precise value of $T_{\mathrm{t}}$ (and $T_{\mathrm{LCST}}$ ) depends on the identity of amino acid $\mathrm{X}^{10}$ and the 
(number average) molecular weight $\left(M_{\mathrm{n}}\right)$ of the polypeptide; ${ }^{13}$ it is further affected by external factors such as the pressure, ${ }^{8}$ the $\mathrm{pH}$ of the solution, ${ }^{19}$ and concentration of other species..$^{20}$ ELP macromolecules are biocompatible, biodegradable, and nonimmunogenic, ${ }^{15,21}$ retaining their transition behavior when fused to other proteins. ${ }^{22}$ These properties make ELPs eminently suitable for applications such as drug delivery, ${ }^{23}$ tissue engineering, ${ }^{21}$ and protein purification. ${ }^{22}$

It is apparent from experimental and modelling studies that aqueous solutions of ELPs undergo intramolecular conformational changes similar to protein folding and exhibit phase separation as the temperature is increased above $T_{\mathrm{t}}$. Several authors have proposed that ELPs exist as two-state systems in water: a folded (collapsed) state and an unfolded (expanded) state, depending on the conditions. ${ }^{7,8,11,24}$ Circular dichroism $(\mathrm{CD})^{7,8,10,12,16,24}$ and Fourier-transform infrared (FT-IR) ${ }^{7,8}$ spectroscopy have lead to the suggestion that there is an increase in the secondary protein structure of ELPs with increases in temperature. These phase transitions are also apparent from thermodynamic measurements of the heat capacity and thermal-expansion coefficient. ${ }^{7,8}$ Molecular simulations have been undertaken to confirm these conformational changes, predicting a decrease in the number of ELP-water hydrogen bonds and a decrease in the number of water molecules in the first hydration shell of the ELP chain with increasing temperature. ${ }^{11,17,18}$ These observations are consistent with the view that the ELP is in a more collapsed state above than below $T_{\mathrm{t}}$. In addition to the aforementioned changes in the intramolecular conformation, turbidity measurements indicate that ELPs aggregate in water to form a coacervate phase (i.e., a viscous gel-like ELP-rich phase $\left.{ }^{12}\right)$ when heated above $T_{\mathrm{t}}{ }^{10,12-14}$ The conformational changes in ELPs thus concur with liquid-liquid phase separation: the collapsed ELP state is associated with a two-phase region of immiscibility (consisting of an essentially pure-water phase in coexistence with an ELP-rich phase); the expanded ELP state is associated with a single-phase region. These transitions are fully reversible. ${ }^{8,10,24}$ Urry and co-workers ${ }^{10,12,14}$ found that these phase transitions provide insight into the fundamental mechanisms that drive protein folding and function.

In our current paper, we use a well-established thermodynamic model, the statistical associating fluid theory for systems characterized by variable range square-well interactions (SAFT-VR SW) ${ }^{25,26}$ parametrized using experimental data at ambient pressures, to represent the global fluid-phase behavior of aqueous solutions of ELPs over broad ranges of pressure, temperature, and concentration. Within the SAFT-VR SW approach, ${ }^{25,26}$ the molecules are treated as chains of square-well (SW) spherical segments with repulsive hard cores and attractive contributions to account for: (a) dispersion interactions; and (b) short-range anisotropic attractive interactions like hydrogen bonding (a pictorial representation is provided in the ESI\$ Fig. S1). These models are conceptually similar to sticky-site models. ${ }^{27}$ Liquid-liquid equilibrium (LLE) data of water + (VPGVG) ${ }_{n}$ for various values of $n$ at ambient pressure $(0.1 \mathrm{MPa})$ are used to parametrize the model; the full parametrization procedure is described in ref. 28. Thermodynamic equations of state (EOSs),
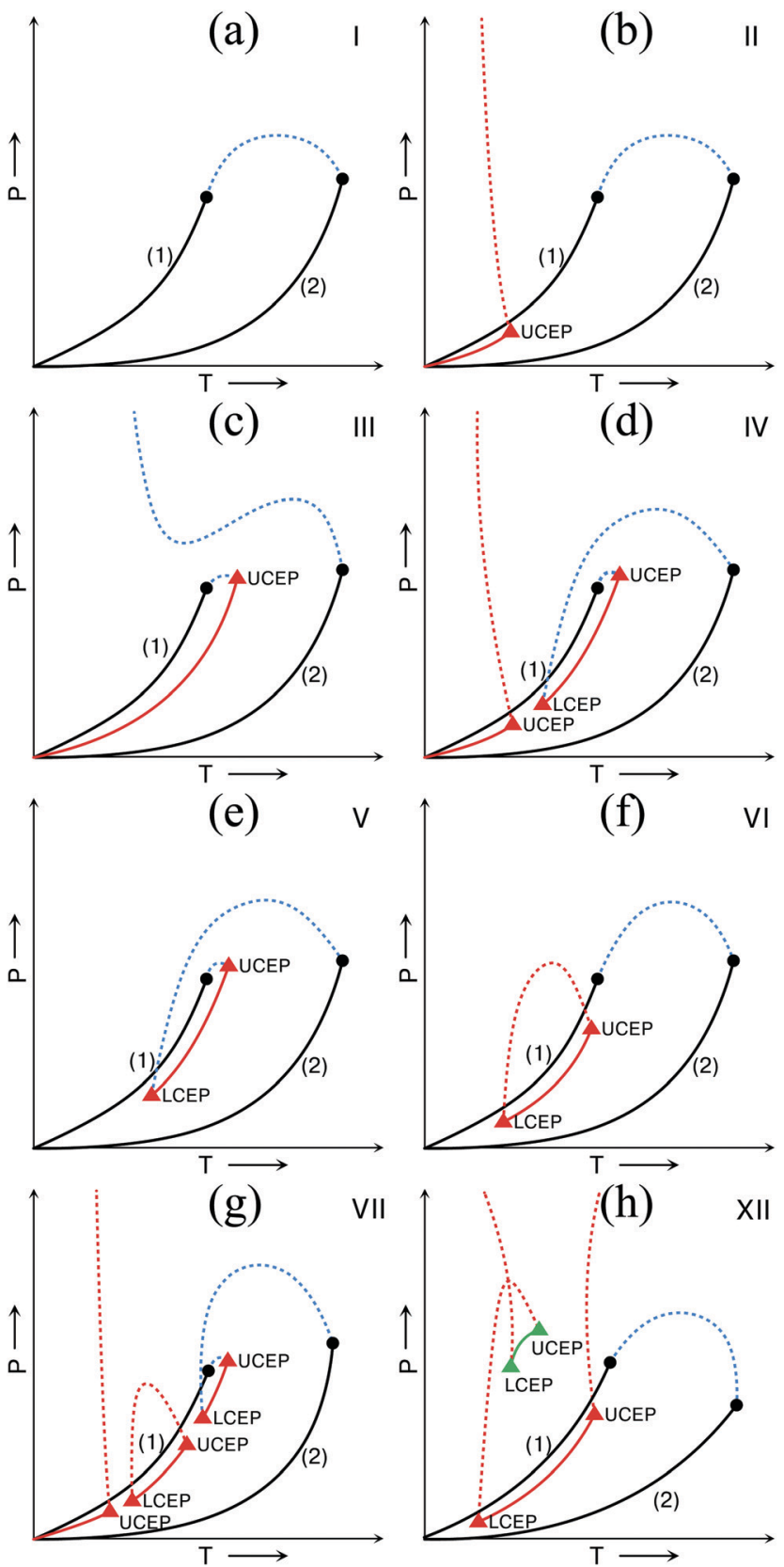

Fig. 1 Pressure-temperature $P-T$ projection schematics of the various types of fluid-phase behavior for binary mixtures. Types I-V were originally proposed by Scott and van Konynenburg. ${ }^{32,33}$ The classification of Type VI was introduced by Rowlinson and Swinton. ${ }^{34}$ Type VII was postulated by Boshkov. ${ }^{37}$ We classify the global phase diagram obtained in our current work for water + (VPGVG) 30 as Type XII. The continuous black curves represent the vapor pressures of the pure components, and the circles represent the pure-component critical points. The loci of the vapor-liquid critical points of the mixture are depicted as blue dashed curves. The continuous red curves represent the three-phase vapor-liquid-liquid equilibrium (VLLE) with the corresponding upper and lower critical end-point temperatures (UCEP and LCEP, respectively) that bound the VLLE marked as red triangles. The continuous green curve represents the liquid-liquid-liquid equilibrium (LLLE) predicted for type XII, with the green triangles denoting the UCEP and LCEP points that bound the LLLE. The red dashed curves represents the loci of lower and upper critical solution temperatures (LCSTs and UCSTs, respectively). 
like SAFT, allow for a direct representation of fluid-phase equilibria, facilitating the study of phase behavior over a broad range of conditions. This enables the determination of the global phase behavior, usually presented as pressure-temperature projections. $^{29-31}$

Following the seminal classification of Scott and van Konynenburg, ${ }^{32,33}$ binary mixtures typically exhibit one of six types of fluid-phase behavior in temperature-pressure-composition space. The pressure-temperature $(P-T)$ projections of the six types of binary phase behavior are shown in Fig. 1(a)-(f). As can be seen from Fig. 1, the classification relies on the topology of the critical lines, which delimit the single-phase and two-phase regions of the mixture. The complexity ranges from systems exhibiting simple vapor-liquid equilibrium (Type I), to systems exhibiting regions of low-temperature LLE (Types II and VI), and systems where the liquid-liquid critical points and vaporliquid critical points are in close proximity giving rise to equilibria between liquid and supercritical phases (Types III, IV and V). The six types of fluid phase behavior following the classification of Scott and van Konynenburg ${ }^{32,33}$ have been observed experimentally. ${ }^{34-36}$ Solvent-polymer systems are often found to exhibit LLE with upper and/or lower critical solution temperatures (UCSTs and/or LCSTs, respectively). ${ }^{34}$ This corresponds to Types II, IV, or V behavior, although Type VI behavior has also been seen for associating polymer systems. ${ }^{34}$ The six types of phase behavior have now been observed with a variety of EOSs, ${ }^{30,31,35-39}$ validating the classification proposed by Scott and Van Konynenburg originally based on an analysis with the van der Waals EOS. There has also been some debate about the existence of other types of fluid-phase behavior predicted using different EOSs and a number of new types have been postulated: VII, ${ }^{37}$ VIII, ${ }^{35}$ IX and X, ${ }^{40}$ and XI. ${ }^{36}$ Of these proposed types, there is experimental evidence supporting Type VII (shown in Fig. 1(g)) in ionic liquid solutions. ${ }^{41}$ The physical reality of the other proposed types (VIII-XI) remains unconfirmed. ${ }^{35,36,40,42}$

The SAFT-VR SW ${ }^{25,26}$ EOS has been shown to capture and predict all six types of phase behavior classified by Scott and van Konynenburg. ${ }^{32,33}$ The global fluid-phase behavior of a variety of systems can be accurately represented over the entire pressure-temperature-composition space with this approach. McCabe and co-workers used SAFT-VR SW to predict: Type I behavior for $n$-hexane $+n$-tetradecane; ${ }^{29}$ Type $\mathrm{V}$ behavior in methane $+n$-hexane; ${ }^{43}$ and Types II and III phase behavior in perfluoro- $n$-alkane $+n$-alkane mixtures $;{ }^{38}$ all in good agreement with experiment. Paricaud et al. have shown transitions from Type I to $\mathrm{V}$ behavior, ${ }^{30}$ and Type IV to III behavior $^{39}$ with increasing disparity in the sizes of the components in the mixture. A similar change in the global behavior from Types II to III through to Type IV with increasing chain length, in agreement with experiment, was demonstrated by Galindo and Blas $^{44}$ for carbon dioxide $+n$-alkane mixtures. For mixtures of associating hydrogen-bonding fluids: Valtz et al., ${ }^{45}$ and dos Ramos et al. $^{46}$ reproduced the Type III global phase behavior exhibited by water + carbon dioxide; and Clark et al. ${ }^{31}$ reproduced the Type VI behavior observed experimentally for the solvent-polymer system water + polyethylene glycol (PEG).
In our current work we use the same SAFT-VR SW approach to predict the high-pressure fluid-phase behavior of aqueous solutions of the ELP (VPGVG) $)_{30}\left(M_{\mathrm{n}}=12302 \mathrm{~g} \mathrm{~mol}^{-1}\right)$. The EOS allows one to extrapolate the phase behavior at known (ambient) conditions to a broad range of temperatures, pressures, and compositions, and thus classify the global phase behavior of the ELP solution. This is often impractical to do experimentally and/ or by direct molecular simulation. We use the SAFT calculations to show that at "lower" pressures $(<50 \mathrm{MPa})$, the phase diagram of water + (VPGVG) $)_{30}$ conforms to Type VI behavior (see Fig. 1(f)), which is also exhibited by other hydrogen-bonding solventpolymer systems such as water + PEG. $^{31}$ At higher pressures (50-155 MPa), we predict a region of LLE bounded by multiple minima (LCSTs), corresponding to so-called bimodal critical behavior. ${ }^{47}$ At even more elevated pressures (>155 $\mathrm{MPa}$ ), two separate LLE regions are predicted, each exhibiting re-entrant closed-loop phase behavior, each bounded at high temperatures by a UCST and at low temperatures by an LCST. As our predicted high-pressure fluid-phase behavior is topologically distinct from all of the previously proposed types of behavior (I-XI), ${ }^{32,33,35-37,40}$ we refer to it as Type XII.

Type XII global phase behavior has not been observed before our current study. A schematic of the $P-T$ projection of a Type XII mixture is depicted in Fig. 1(h). Our work is the first offlattice calculation to predict bimodal critical behavior, which has been observed experimentally for other binary solvent-polymer systems at near-atmospheric pressures; ${ }^{48-54}$ the bimodal behavior has been represented using lattice-based theories, but pressure effects cannot be described using this type of incompressiblelattice approaches. ${ }^{54-56}$ As far as we are aware, multiple re-entrant regions of LLE have not been observed or predicted before for binary systems. The presence of bimodal critical behavior and two separate regions of closed-loop LLE means that there are multiple stable liquid phases for different ranges of the concentration of the ELP. Experimental studies suggest that at ambient pressure the phase separation of water + ELP is accompanied by conformation changes in the ELP macromolecules. ${ }^{7,8,10-14,24}$ The multiple stable liquid phases containing the ELP molecules predicted here at high pressure may therefore represent different conformations of the ELP macromolecule (than the two conformations found at ambient conditions). In the pressure-temperature $(P-T)$ isopleths at fixed composition of the polypeptide, the liquid-liquid critical boundaries of water + ELP resemble those of protein denaturation diagrams, further strengthening the relevance of the behavior of ELPs to proteins.

\section{Computational methods}

The statistical associating fluid theory with attractive potentials of variable-range (SAFT VR SW) ${ }^{25,26}$ is used in our current work. In the SAFT approach molecules are treated as chains of homonuclear hard-sphere segments with dispersion interactions represented with SW potentials. Segments can incorporate additional short-range anisotropic association sites to account 
for hydrogen bonding (modeled using short-range off-centre SW potentials).

Details of the parametrization procedure for aqueous solutions of (VPGVG $)_{n}$ can be found in ref. 28. The model parameters were refined to a combination of data from experiment ${ }^{13}$ and molecular simulation, both at $0.1 \mathrm{MPa}$. A visual representation of the SAFT model and the number of association sites on each segment can be seen in ESI, $\ddagger$ Fig. S1. The water segments and the segments of the ELP chain are characterised by different interaction potentials. Each amino acid in the ELP is modeled as a single spherical segment with the same SW interaction, the distinguishing feature between amino acids being the number of association sites. The terminal amino acids are modeled with a different number of association sites from the amino acids along the backbone of the chain. The association sites only interact with sites representing an opposite charge. The unlike (between water and ELP groups) interaction parameter was refined to experimental LLE data for aqueous solutions of (VPGVG) molecules for a series of molecular weights $(n=30,60,90$, and 120) at ambient pressure $0.1 \mathrm{MPa} .{ }^{13}$ The parameters were estimated from data over a relatively small temperature $(\sim 295-345 \mathrm{~K})$ and composition $\left(w_{\mathrm{ELP}} \sim 0.00-0.03\right)$ range. The SAFT-VR SW formalism is (a)

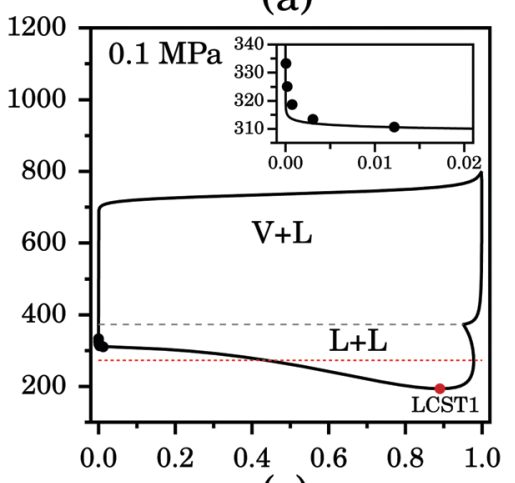

(c)
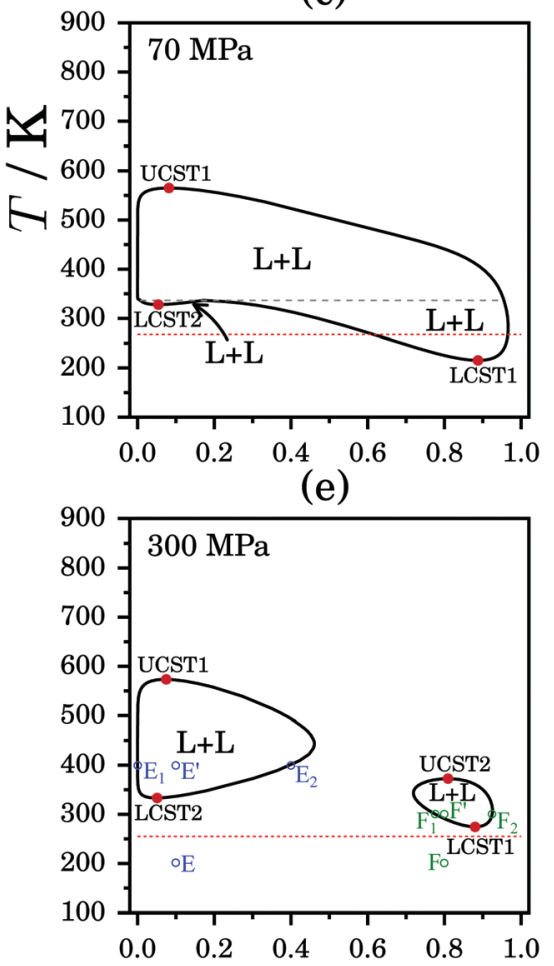

(b)

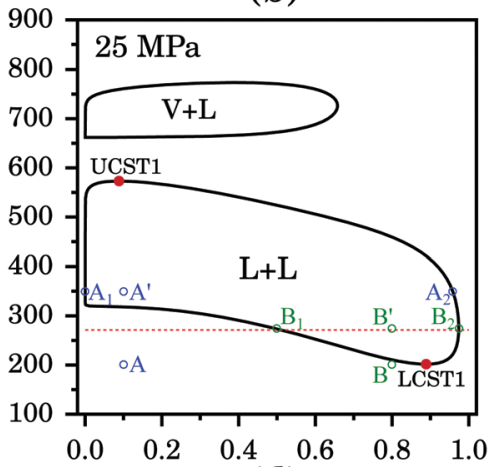

(d)
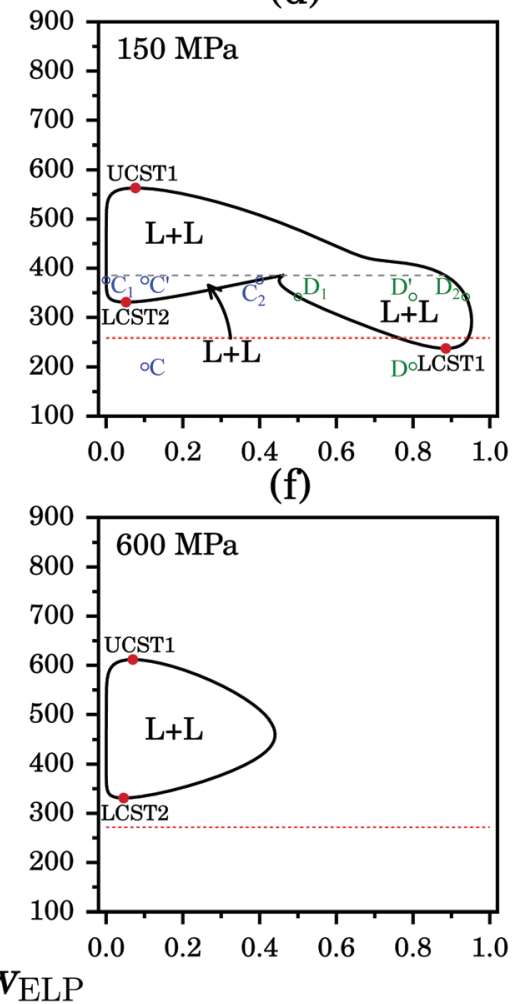

Fig. 2 Temperature-mass fraction ( $T-W_{E L P}$ ) isobars of the fluid-phase equilibria of aqueous solutions of (VPGVG) 30 at (a) $0.1 \mathrm{MPa}$, (b) $25 \mathrm{MPa}$, (c) $70 \mathrm{MPa}$, (d) $150 \mathrm{MPa}$, (e) $300 \mathrm{MPa}$, and (f) $600 \mathrm{MPa}$. The experimental data ${ }^{13}$ (filled black circles) are visible in the inset plot in (a), highlighting the accuracy of the SAFT calculations at $0.1 \mathrm{MPa}$. The continuous black curves denote the phase envelope calculated using the SAFT-VR SW EOS. The grey dashed lines represent either the vapor-liquid-liquid (VLLE) three-phase line (at 0.1 MPa (a)) or the liquid-liquid-liquid (LLLE) three-phase line (at $70 \mathrm{MPa}$ (c) and $150 \mathrm{MPa}(\mathrm{d})$ ). The filled red circles represent the calculated UCSTs and LCSTs. The red dashed lines represent the experimental melting point of pure water. ${ }^{58}$ The experimental error bars are omitted as they are smaller than the symbols. The hollow blue and green circles are labelled points of interest referred to in the text. The labels $L$ and $V$ denote liquid and vapor phases, respectively. 
then used to extrapolate the fluid-phase behavior over a broad range of conditions. The resulting parameters are state independent, as demonstrated in previous work ${ }^{31,39}$ in which we have studied the phase behaviour of polymer + solvent mixtures using the SAFT-VR approach over wide ranges of thermodynamic conditions, including high-pressures.

As with other thermodynamic perturbation theories, SAFTVR SW is expressed in the form of the Helmholtz free energy. The other thermodynamic properties (such as the pressure and chemical potential) are obtained from standard thermodynamic identities. The phase equilibria are evaluated at fixed pressure, temperature, and mixture composition using the Helmholtz free energy Langrangian dual (HELD) algorithm, ${ }^{57}$ which has been shown to accurately resolve the Gibbs free energy minima. A series of isobars are produced to determine the critical points of water $+(\mathrm{VPGVG})_{30}$. The nature of each phase is determined from an analysis of the packing fraction.

\section{Results \& discussion}

\subsection{Temperature-ELP mass fraction $\left(T-w_{\text {ELP }}\right)$ isobars}

Predicted constant-pressure temperature-mass fraction ( $\left.T-w_{\mathrm{ELP}}\right)$ slices (isobars) of the fluid-phase equilibrium for water + $(\text { VPGVG })_{30}$ are shown in Fig. 2, in which a dramatic change in the behavior with pressure can be seen. The nature of each phase is determined from the packing fraction $\eta$ (the fraction of the total volume occupied by the molecules). The liquid phase is identifiable for $\eta>0.1$. In ESI, $\ddagger$ Fig. S2, the $T-\eta$ diagrams are shown for the corresponding $T-w_{\mathrm{ELP}}$ isobars depicted in Fig. 2 . The experimental water $+(\mathrm{VPGVG})_{30}$ data at $0.1 \mathrm{MPa},{ }^{13}$ which is used to develop the interaction parameters of the model, ${ }^{28}$ is accurately reproduced by the SAFT-VR SW approach (see the inset plot in Fig. 2(a)). The behavior observed for the $0.1 \mathrm{MPa}$ isobar is typical of a water + polymer system with an LCSTbounded (LCST1) LLE region at lower temperatures and a VLE region at higher temperatures. ${ }^{30}$ Vapor-liquid-liquid coexistence (VLLE) is observed at the intersection of the LLE and VLE regions. There is a second water-rich VLE region just below the three-phase line, but it is negligibly small for the relatively high molecular weight (compared to the solvent) of the ELPs. ${ }^{28}$ From a comparison of the phase diagrams at $0.1 \mathrm{MPa}$ (Fig. 2(a)) and $25 \mathrm{MPa}$ (Fig. 2(b)), it is apparent that the VLE region shrinks with increasing pressure. Above 9.4 MPa no three-phase coexistence is observed as the VLE and LLE regions have separated, and the LLE region becomes bounded by a UCST (UCST1). For pressures above $40.3 \mathrm{MPa}$, the system is above the maximum in the vaporliquid critical line and the VLE region ceases to exist altogether, as seen from the $70 \mathrm{MPa}$ isobar in Fig. 2(c). The LLE region is less sensitive to pressure (compare Fig. 2(b) and (c)), but also slowly decreases in size, as the LCST (LCST1) increases with increasing pressure. At 48.6 MPa a second LCST (LCST2) appears at low concentration of the polypeptide. Although the LLE region associated with LCST2 is at first relatively small, it widens in composition with increasing pressure; this region is clearly seen at 70 and $150 \mathrm{MPa}$ in Fig. 2(c) and (d), respectively. The three LLE regions, bounded by the LCST1, LCST2, and UCST1 (see Fig. 2(c) and (d)), intersect at a three-phase liquid-liquid-liquid coexistence (LLLE) temperature. As the pressure is increased, the extent of the LLE region bounded by the LCST1 continues to decrease in both composition and temperature, whereas the $T-w_{\text {ELP }}$ region associated with LCST2 expands. At around $155 \mathrm{MPa}$ one can first see two separate closed-loop regions with a second UCST (UCST2); these are clearly apparent in the $300 \mathrm{MPa}$ isobar (Fig. 2(e)). The UCST2 and LCST1, which bound the closedloop at high concentrations of ELP, continue to converge with increasing pressure, and the region disappears at $\sim 500 \mathrm{MPa}$ (between Fig. 2(e) and (f)). The UCST1 and LCST2, encompassing the closed-loop region of low ELP concentration, slowly diverge as the pressure is increased.

The phase with intermediate concentrations of $\operatorname{ELP}(e . g$., points C2 and E2 from Fig. 2), which is in equilibrium with essentially pure water almost-pure water and is related to the LCST2-bound LLE region seen at high pressures, will likely contain ELP molecules with a different conformation than those found at lower pressures. At low pressure and low ELP concentration (point A in Fig. 2(b)), the ELP is predicted to be fully soluble in water below $T_{\mathrm{t}}$. According to experiment and molecular simulation the ELP at these conditions (point A) is in its expanded conformation. ${ }^{7,8,11,14,24}$ At the same overall composition above $T_{\mathrm{t}}$ (point $\mathrm{A}^{\prime}$ in Fig. 2(b)), the system demixes into an essentially pure-water phase (labelled $\mathrm{A}_{1}$ ) and a phase with a high ELP concentration (labelled $\mathrm{A}_{2}$ ). Phase $\mathrm{A}_{2}$ is expected to be a coacervate phase $\mathrm{e}^{10,12-14}$ (i.e., a viscous gel-like ELP-rich phase ${ }^{12}$ ); the general consensus is that the ELP is in a collapsed state in this phase. ${ }^{7,8,11,14,24}$ This high-ELP concentration phase persists up to pressures of $\sim 500 \mathrm{MPa}$ (see points B2, D2, and F2). The LCST2-bounded LLE region, first appearing at around 48.6 MPa and clearly visible at $150 \mathrm{MPa}$ in Fig. 2(d) at low ELP concentrations (point $\mathrm{C}^{\prime}$ ), corresponds to an equilibrium between an essentially pure-water phase (phase $\mathrm{C}_{1}$ ) and a phase of intermediate ELP concentration $\left(\mathrm{C}_{2}\right)$ that persists to higher pressures (point E2 in Fig. 2(e)). We postulate that the demixing seen at $\mathrm{C}^{\prime}$ and at $\mathrm{E}^{\prime}$ is driven by a change in the ELP conformation with pressure. The large difference in the water content between phases with intermediate ELP concentrations (C2 and E2) and phases with high ELP concentrations (A2, B2, D2, and F2) means that the ELP is likely to have a different conformation in these two types of phases. We hypothesize that the ELP is in a partiallycollapsed state in phases C2 and E2.

\subsection{Pressure-temperature $(P-T)$ projection}

The fluid-phase behavior for water $+(\text { VPGVG })_{30}$ is summarized as a global pressure-temperature $(P-T)$ projection in Fig. 3(a), in which the pure-component vapor-pressure curves $\left(P_{\mathrm{v} \text {,water }}\right.$ and $P_{\mathrm{V}, \mathrm{ELP}}$ ), the liquid-liquid critical lines (corresponding to LCST1, LCST2, UCST1, UCST2), the loci of the vapor-liquid critical points of the mixture $(T, P)_{\mathrm{c}, \mathrm{mix}} \cdot$ ), and the trends of the three-phase coexistence lines (VLLE and LLLE) are presented. A schematic of the $P-T$ projection is also presented in Fig. 1 (labelled Type XII), and the full $P-T-w_{\mathrm{ELP}}$ surface can be found in ESI, $\neq$ Fig. S3 and S4. Note that since the vapor pressure of ELP $P_{\mathrm{v}, \mathrm{ELP}}$ is essentially 
negligible (Fig. 3(b)), the VLLE line virtually coincides with the vapor pressure $P_{\mathrm{v} \text {,water }}$ (Fig. $3(\mathrm{c})$ ).

The global fluid-phase behavior of water $+(\text { VPGVG })_{30}$ depicted as a $P-T$ projection in Fig. 3(a) is characterized by a type of liquid-liquid phase behavior which does not conform to the existing classifications for binary mixtures (Types I-XI). We thus propose to classify the global phase diagram with the general topology shown in Fig. 3(a) as Type XII. Below 48.6 MPa, the $P-T$ projection shown in Fig. 3(a) closely resembles Type VI fluid-phase behavior (see Fig. 1(f)) with a characteristic solventpolymer VLE region (see Fig. 3(c)), where the vapor pressure of the ELP $P_{\mathrm{v}, \mathrm{ELP}}$ is very low (see Fig. 3(b)) and the lower and upper critical end points (LCEP1 and UCEP1) bounding the closedloop LLE region are very close to the vapor-pressure of pure water $P_{\mathrm{v} \text {,water }}$ (see Fig. $3(\mathrm{c})$ ). This is not surprising, as type VI fluid-phase behavior is often exhibited by other associating solvent + polymer systems. $^{31}$ The phase diagram of Fig. 3(a) starts to differ markedly from Type VI behavior at $48.6 \mathrm{MPa}$ : a second lower critical end point (LCEP2, marking the first appearance of LCST2) and a three-phase LLLE line are predicted at $\sim 50 \mathrm{MPa}$; furthermore, a new upper critical end point (UCEP2) is observed at $155 \mathrm{MPa}$ where the LLLE terminates and the LLE region splits into two separate closed-loop regions (as seen in Fig. 2(e)). This type of high-pressure fluid-phase equilibria is distinct from previously-reported global types of phase behavior (Types I-XI), ${ }^{32,33,35-37,40}$ which do not include LLE regions bounded by multiple LCSTs or UCSTs (bimodal behavior), LLLE coexistence (Fig. 2(c) and (d)) or multiple closed-loop regions of LLE (Fig. 2(e)). As shown schematically in Fig. 1(h), the Type XII phase behavior found for water + $(\mathrm{VPGVG})_{30}$ behavior is characterized by an LCST and a UCST emanating from within the LLE region, which sets it apart from that of Type I-VI systems. ${ }^{32,33,37}$
Type XII phase behavior also differs from that of Types VIII-X proposed by van Pelt and co-workers ${ }^{35,36,40}$ using the simplifiedperturbed-hard-chain theory (SPHCT), as Types VIII-X are characterized by additional liquid critical points in the proximity of the binary vapor-liquid critical line rather than within the LLE region as seen with Type XII. Type XI predicted by van Pelt ${ }^{36}$ using SPHCT only contains a single LLE region with critical end points originating at negative pressures, and is thus also distinct from Type XII proposed here.

\subsection{Bimodal liquid critical point behavior}

The LLE behavior with multiple LCSTs or UCSTs (referred to as bimodal behavior) that we predict for water $+(\text { VPGVG })_{30}$ (Fig. 2(c) and (d)) has been observed experimentally for other solvent-polymer mixtures. The first documented bimodal UCST and LCST behavior in solvent-polymer systems was for acetone + PS mixtures (polydispersity $M_{\mathrm{w}} / M_{\mathrm{n}}=1.03$, where $M_{\mathrm{w}}$ is the weight average molecular weight) between 0.35 and $2.0 \mathrm{MPa}{ }^{50}$ These pressures are relatively low compared to the pressures at which this type of behavior is observed in our study for water + (VPGVG $)_{30}$. Bimodal LCSTs have also been observed experimentally in water + poly(vinyl methyl ether) (PVME) mixtures ${ }^{51-54}$ with polydispersities ranging from $M_{\mathrm{w}} / M_{\mathrm{n}}=1.10$ to 7.8. ${ }^{53}$ The experiments for water + PVME were undertaken only at atmospheric pressure, so that an assessment of the global phase behavior cannot be made. Although bimodal behavior can in some cases be attributed to polydispersity, ${ }^{49}$ the aforementioned experiments can lead one to suggest that this behavior persists at low polydispersity. ${ }^{50,53}$

Thermodynamic lattice-based models have been used to reproduce and/or predict the bimodal LCSTs and UCSTs seen experimentally in solvent-polymer systems. Flory-Huggins-Staverman
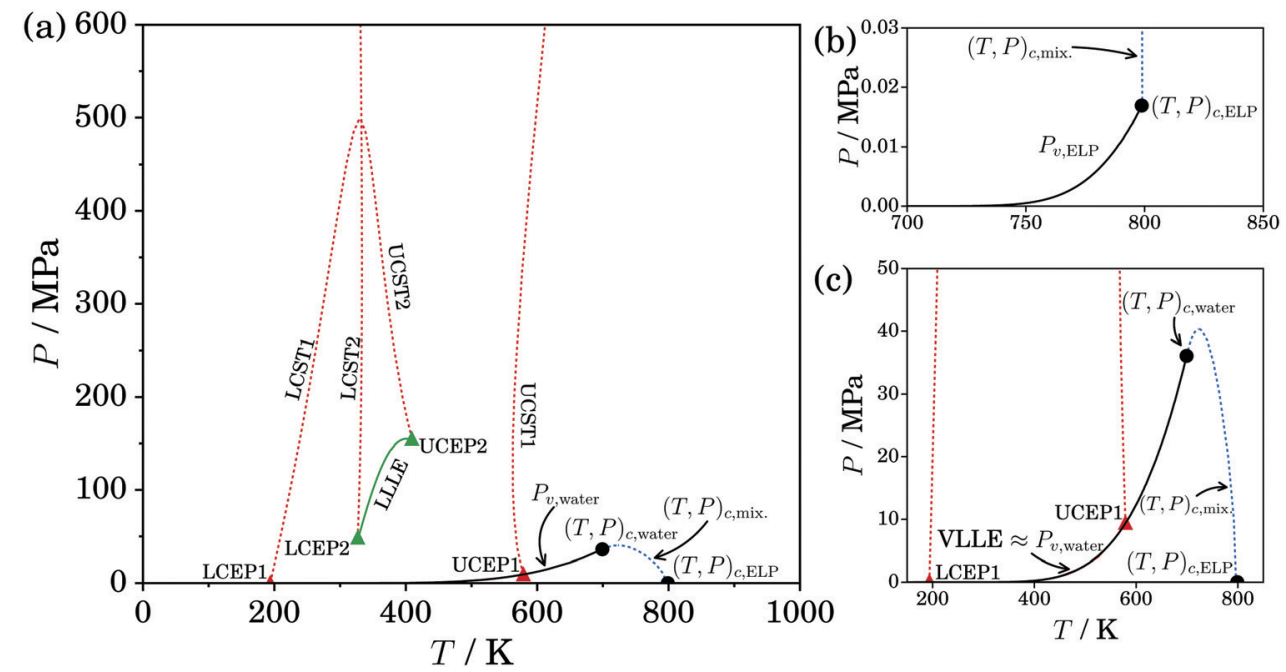

Fig. 3 Pressure-temperature $(P-T)$ projection of global phase behavior of water + (VPGVG) 30 predicted with SAFT-VR SW: (a) the complete phase diagram; (b) the vapor-pressure curve of the ELP; and (c) the closed-loop Type-VI like behavior. The continuous black curves represent the vapor pressures of the pure components, $P_{\mathrm{v}, \text { water }}$ and $P_{\mathrm{v}, \mathrm{EL} L \mathrm{P}}$ being the lower curve (highlighted on plot (b)). The black circles represent the pure component

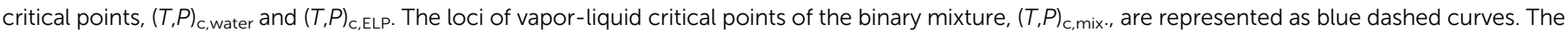
three-phase vapor-liquid-liquid equilibrium (VLLE) line is bound by the UCEP1 and LCEP1 represented as red triangles. The continuous green curve represents the liquid-liquid-liquid equilibrium (LLLE), with the green triangles denoting the UCEP2 and LCEP2 points that bound this curve. The red dashed curves represent the loci of the liquid critical points (LCST1, LCST2, UCST1 and UCST2). 
(FHS) theories rely on state-dependent $(\chi)$ parameters to describe the experimentally observed phase behavior of acetone $+\mathrm{PS}^{55}$ and water $+\mathrm{PVME}^{53}$ mixtures. The Wertheim lattice thermodynamic pertubation theory (Wertheim-LTPT) was the first theory used to predict bimodal LCSTs ${ }^{54,56}$ and the existence of multiple lowtemperature UCSTs. ${ }^{54,56}$ Unlike the experimental systems, however, all the aforementioned theoretical studies (including our current work) treat the polymer as monodisperse, which suggests that the multiple LCSTs and UCSTs are not a consequence of polydispersity. Our work with SAFT-VR SW represents the first use of an off-lattice approach to predict this kind of bimodal behavior. Anisotropic association interactions such as hydrogen bonding are the key to closed-loop LLE behavior. ${ }^{34,59}$ The fact that both the Wertheim-LTPT and our continuum SAFT approach, which is based on the work by Wertheim ${ }^{60}$ on fluids with short-range anisotropic interactions, can be used to predict bimodal LCST behavior implies that hydrogen bonding also plays an important role in this phenomena. A further major advantage of continuum EOS approaches such as SAFT is that one can account for changes in the phase behavior with pressure without the introduction of pressure-dependent parameters.

\subsection{Pressure-temperature $(P-T)$ constant composition $\left(w_{\mathrm{ELP}}\right)$}

The constant composition $P-T$ phase diagrams (isopleths) for water $+(\text { VPGVG })_{30}$ predicted with SAFT-VR SW at $w_{\text {ELP }}=0.10$, $0.40,0.50$, and 0.80 in Fig. $4(\mathrm{~b})-(\mathrm{e})$ bear similarities to the "generic" protein temperature-pressure denaturation diagrams, ${ }^{6,61-63}$ shown schematically in Fig. 4(a). The behavior depicted in Fig. 4(a) corresponds to low-protein concentrations of less than $1 \%$ by weight. ${ }^{6,63}$ In order to help understand the $P-T$ diagrams in Fig. 4, the points of relevance labelled in the isobaric slices of the fluid-phase equlibria in Fig. 2 are also shown. As a point of reference, the total protein content of a cell is $\sim 20 \%$ by weight. ${ }^{64}$ Although Fig. $4(\mathrm{c})-(\mathrm{e})$ correspond to aqueous solutions with ELP mass fractions in excess of the protein mass fractions typically found in cells, our predictions still allow for qualitative comparisons to the protein denaturation diagram depicted in Fig. 4(a). We should also note at this stage that the phase behavior is highly dependent on the molecular weight of the $\operatorname{ELP}^{28}$ (see ESI, $\ddagger$ Fig. S5). For an overall ELP concentration of $w_{\text {ELP }}=0.80$, corresponding to Fig. $4(\mathrm{e})$, the $P-T$ phase boundaries closely resemble the "generic" protein denaturation diagram of Fig. 4(a). The points labelled in Fig. 4(e) $\left(\mathrm{B}^{\prime}, \mathrm{D}^{\prime}\right.$, and $\left.\mathrm{F}^{\prime}\right)$ all correspond to the demixed LLE region bounded by LCST1 with an equilibrium between a highconcentration ELP coacervate phase, in which the polypeptide chain is in a collapsed state, and a phase with intermediate ELP concentration. As the overall concentration of ELP is lowered to $w_{\text {ELP }}=0.50$ (see Fig. $4(\mathrm{~d})$ ), the $P-T$ diagram is characterized by two peaks: the lower-temperature peak corresponds to the LLE region associated with LCST1, and the higher-temperature peak corresponds to the LLE region associated with LCST2; in the latter there is coexistence between an essentially pure-water phase and a phase with an intermediate concentration of ELP. On decreasing the overall ELP concentration from $w_{\mathrm{ELP}}$ of 0.50 to 0.40 (Fig. 4(d) to Fig. 4(c)) one can observe a drastic change in (a)

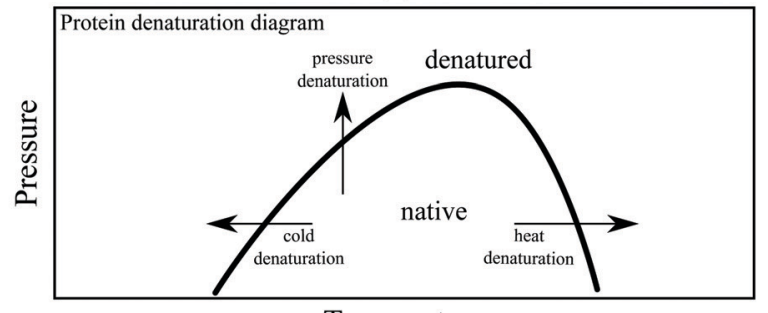

(b)
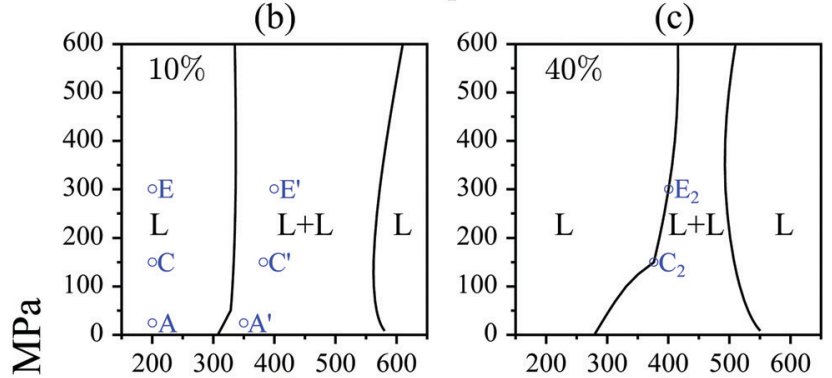

(d)

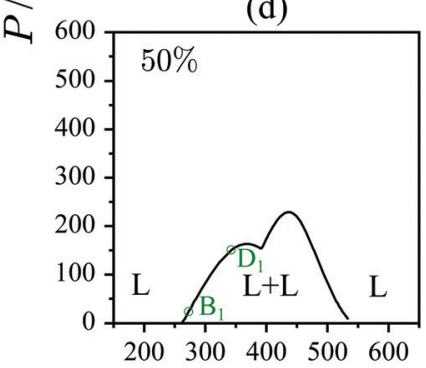

(e)

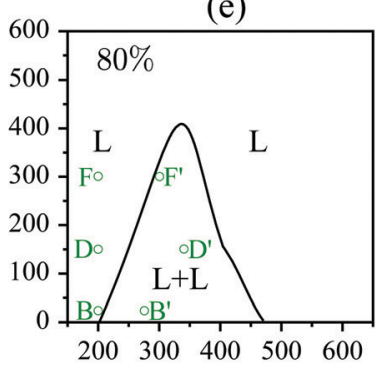

$T / \mathrm{K}$

Fig. 4 Pressure-temperature $(P-T)$ phase diagrams at constant ELP concentration (isopleths). (a) Schematic of a "generic" protein denaturation diagram ${ }^{6,61-63}$ at low protein-concentration $\left(w_{\text {protein }}<0.01\right)$. The predicted phase boundaries obtained with SAFT-VR SW for water + (VPGVG) 30 at mass fractions of: (b) $w_{E L P}=0.10$; (c) $w_{E L P}=0.40$; (d) $w_{E L P}=0.50$; and (e) $w_{E L P}=$ 0.90. The hollow blue and green circles are labelled points of interest mentioned in the main text, and correspond to those with the same label and color in Fig. 2.

the phase behavior: the high-temperature peak previously associated with LCST2 in Fig. 4(d) opens upwards giving rise to the chimney-shaped phase boundary seen in Fig. 4(c). At the lower overall ELP concentration of $w_{\mathrm{ELP}}=0.10$ (Fig. 4(b)), the high concentration ELP phase is only present in the demixed state at low pressure (point $\mathrm{A}^{\prime}$ ), with the higher-pressure states (points $\mathrm{C}^{\prime}$ and $\mathrm{E}^{\prime}$ ) corresponding to demixed states with intermediate concentrations of ELP (also seen in Fig. 4(c)). The phase diagrams seen in Fig. 4(b)-(d) are markedly different from the "generic" schematic of the protein denaturation diagram (Fig. 4(a)). These differences are caused by the presence of the LLE region associated with LCST2.

The phases with intermediate concentrations of ELP associated with LCST2 may correspond to so-called molten globules in analogy with proteins. The LLE associated with LCST2 found at high pressures corresponds to an equilibrium between a phase which is essentially pure water and a phase with an intermediate concentration of ELP (points C2 and E2 in Fig. 2(d) and (e)). 
We propose that in the phases with intermediate ELP concentrations (C2 and E2) found at these elevated pressures, the polypeptide is in a partially-collapsed state. Partially collapsed (or unfolded) states have also been found experimentally for proteins and are referred to as molten globules. ${ }^{65,66}$

\section{Conclusions}

We use a statistical thermodynamics approach, SAFT-VR $\mathrm{SW},{ }^{25,26}$ to predict the global fluid-phase behavior of aqueous solutions of $(\mathrm{VPGVG})_{30}$. The model ${ }^{28}$ is parameterized to experimental data at $0.1 \mathrm{MPa} .{ }^{13}$ At low pressures, the system exhibits closed-loop phase behavior, which is seen in other hydrogenbonding solvent-polymer systems. ${ }^{31}$ At high pressures (above 48.6 $\mathrm{MPa}$ ), we predict that the phase envelopes of water + $(\text { VPGVG) })_{30}$ are bounded by multiple LCSTs (bimodal LCSTs). Bimodal LLE behavior has been seen experimentally in other solvent-polymer mixtures, ${ }^{50-52}$ and has been described with lattice-based models. ${ }^{53-56}$ Our work represents the first offlattice (continuum) prediction of bimodal LLE behavior. Above $155 \mathrm{MPa}$, we predict the existence of two separate regions of re-entrant liquid-liquid immiscibility in aqueous (VPGVG) $)_{30}$, a behavior that has not been reported previously. The fluid-phase equilibria of the water + ELP system considered here is topologically distinct from the types of global phase behavior proposed by Scott and van Konynenburg, ${ }^{32,33}$ and by others. ${ }^{34-37,40}$ We categorize this as a new type: Type XII.

At high ELP concentration, the $P-T$ phase diagram resembles a "generic" protein denaturation diagram, whereas at low ELP concentrations a different topology in the phase behavior is predicted that may correspond to the presence of "moltenglobule"-like states. Aqueous ELPs are known to undergo intramolecular conformational changes at ambient pressure, as seen with protein cold denaturation, from a collapsed to expanded state as the temperature is decreased to below $T_{\mathrm{t} \cdot}{ }^{7,8,11,24} \mathrm{We}$ find that the $P-T$ diagram at an overall ELP concentration of $w_{\mathrm{ELP}}=0.80$ (see Fig. 4(e)) closely resembles a protein denaturation diagram (shown schematically in Fig. 4(a)). At lower overall concentrations of ELP (Fig. 4(b)-(d)), the $P-T$ diagrams are different from the "generic" protein denaturation diagram (depicted in Fig. 4(a)). The deviations stem from the appearance of the second LCST (LCST2). We hypothesize that this region corresponds to partially-collapsed or, the analogy with proteins, molten-globule states. ${ }^{65,66}$ The prediction of new LLE regions with SAFT-VR SW demonstrates the advantage of using equations of state to study the global fluidphase behavior of aqueous solutions of ELPs as a proxy for more complex polypeptides, like IDPs, ${ }^{9}$ and for proteins in general.

We speculate on the relevance of the high-pressure fluid-phase behavior predicted for water + ELP mixtures to protein systems in general. In the deep sea, organisms thrive at high pressures of 50 to $110 \mathrm{MPa}$ and temperatures of $273 \mathrm{~K}$ up to $395 \mathrm{~K} .^{67,68}$ The overall protein concentration in the cells of these organisms is of the order of $20 \%$ by weight. At these conditions, we predict that the ELP does not appear in its collapsed or the "native" protein state. Instead we find either the fully expanded state (point C in Fig. 2(d)) or a phase of intermediate ELP concentration that we propose corresponds to a partially-collapsed or "moltenglobule" protein state. This may explain the presence of specialised proteins found in deep-sea fish that can withstand these pressures and remain in their native state. ${ }^{67}$ We find that increasing the molecular weight of the ELP at elevated pressure, leads to a decrease in $T_{\mathrm{t}}$ and shrinks the temperature range of the LCST2bound LLE region (see ESI, $\$$ Fig. S5), thereby increasing the temperature range over which the collapsed or "native" state is stable (phase A2 and B2 in Fig. 2(b), D2 in Fig. 2(d), and F2 in Fig. 2(e)). The drastic changes in phase behavior seen when the overall ELP concentration is varied (cf. Fig. 4(b) through Fig. 4(e)) further illustrate that as well as the temperature and pressure, the concentration of polypeptide, a variable not usually considered in protein stability studies, ${ }^{5,6,69}$ strongly affects the nature of the phase (single or demixed state) of the water + ELP mixture at elevated pressures.

\section{Conflicts of interest}

There are no conflicts to declare.

\section{Acknowledgements}

This work was supported in part by the NSF's Research Triangle MRSEC (DMR-1121007), NSF's (CBET-1743432), and the North Carolina State University College of Engineering. This work used the Extreme Science and Engineering Discovery Environment (XSEDE), which is supported by National Science Foundation grant number ACI-1053575. T. L. thanks Imperial College London for the award of a President's PhD scholarship. We also acknowledge financial support from the Engineering and Physical Sciences Council (EPSRC) of the U.K. (grants EP/E016340 and EP/J014958/1) to the Molecular Systems Engineering (MSE) group.

\section{Notes and references}

1 R. M. Hazen, N. Boctor, J. A. Brandes, G. D. Cody, R. J. Hemley, A. Sharma and H. S. Yoder, J. Phys.: Condens. Matter, 2002, 14, 11489-11494.

2 P. H. Yancey, M. E. Gerringer, J. C. Drazen, A. A. Rowden and A. Jamieson, Proc. Natl. Acad. Sci. U. S. A., 2014, 111, 4461-4465.

3 N. D. Gallo, J. Cameron, K. Hardy, P. Fryer, D. H. Bartlett and L. A. Levin, Deep Sea Res., Part I, 2015, 99, 119-133.

4 A. Sharma, Science, 2002, 295, 1514-1516.

5 S. A. Hawley, Biochemistry, 1971, 10, 2436-2442.

6 R. Ravindra and R. Winter, ChemPhysChem, 2003, 4, 359-365.

7 E. Schreiner, C. Nicolini, B. Ludolph, R. Ravindra, N. Otte, A. Kohlmeyer, R. Rousseau, R. Winter and D. Marx, Phys. Rev. Lett., 2004, 92, 148101.

8 C. Nicolini, R. Ravindra, B. Ludolph and R. Winter, Biophys. J., 2004, 86, 1385-1392.

9 S. Roberts, M. Dzuricky and A. Chilkoti, FEBS Lett., 2015, 589, 2477-2486. 
10 D. W. Urry, T. L. Trapane and K. U. Prasad, Biopolymers, 1985, 24, 2345-2356.

11 R. Rousseau, E. Schreiner, A. Kohlmeyer and D. Marx, Biophys. J., 2004, 86, 1393-1407.

12 D. W. Urry, J. Protein Chem., 1988, 7, 1-34.

13 D. E. Meyer and A. Chilkoti, Biomacromolecules, 2004, 5, 846-851.

14 D. W. Urry, J. Phys. Chem. B, 1997, 101, 11007-11028.

15 J. A. Mackay and A. Chilkoti, Int. J. Hyperthermia, 2008, 24, 483-495.

16 H. Nuhn and H.-A. Klok, Biomacromolecules, 2008, 9, 2755-2763.

17 B. Zhao, N. K. Li, Y. G. Yingling and C. K. Hall, Biomacromolecules, 2016, 17, 111-118.

18 J. E. Condon, T. B. Martin and A. Jayaraman, Soft Matter, 2017, 13, 2907-2918.

19 J. A. MacKay, D. J. Callahan, K. N. FitzGerald and A. Chilkoti, Biomacromolecules, 2010, 11, 2873-2879.

20 T. Yamaoka, T. Tamura, Y. Seto, T. Tada, S. Kunugi and D. A. Tirrell, Biomacromolecules, 2003, 4, 1680-1685.

21 D. L. Nettles, A. Chilkoti and L. A. Setton, Adv. Drug Delivery Rev., 2010, 62, 1479-1485.

22 E. E. Fletcher, D. Yan, A. A. Kosiba, Y. Zhou and H. Shi, Protein Expression Purif., 2019, 153, 114-120.

23 J. C. Rodríguez-Cabello, F. J. Arias, M. A. Rodrigo and A. Girotti, Adv. Drug Delivery Rev., 2016, 97, 85-100.

24 H. Reiersen, A. R. Clarke and A. R. Rees, J. Mol. Biol., 1998, 283, 255-264.

25 A. Gil-Villegas, A. Galindo, P. J. Whitehead, S. J. Mills, G. Jackson and A. N. Burgess, J. Chem. Phys., 1997, 106, 4168.

26 A. Galindo, L. A. Davies, A. Gil-Villegas and G. Jackson, Mol. Phys., 1998, 93, 241-252.

27 N. Kern and D. Frenkel, J. Chem. Phys., 2003, 118, 9882-9889.

28 B. Zhao, T. Lindeboom, S. Benner, G. Jackson, A. Galindo and C. K. Hall, Langmuir, 2017, 33, 11733-11745.

29 C. McCabe and G. Jackson, Phys. Chem. Chem. Phys., 1999, 1, 2057-2064.

30 P. Paricaud, A. Galindo and G. Jackson, Mol. Phys., 2003, 101, 2575-2600.

31 G. N. I. Clark, A. Galindo, G. Jackson, S. Rogers and A. N. Burgess, Macromolecules, 2008, 41, 6582-6595.

32 R. L. Scott and P. H. van Konynenburg, Discuss. Faraday Soc., 1970, 49, 87.

33 P. H. van Konynenburg and R. L. Scott, Philos. Trans. R. Soc., A, 1980, 298, 495-540.

34 J. S. Rowlinson and F. L. Swinton, Liquids and Liquid Mixtures, Butterworth, London, 3rd edn, 1982.

35 A. Van Pelt, C. J. Peters and J. De Swaan Arons, J. Chem. Phys., 1991, 95, 7569.

36 A. van Pelt, PhD thesis, Technische Universiteit Delft, 1992.

37 L. Z. Boshkov, Dokl. Akad. Nauk SSSR, 1987, 294, 901-905.

38 C. McCabe, A. Galindo, A. Gil-Villegas and G. Jackson, J. Phys. Chem. B, 1998, 102, 8060-8069.

39 P. Paricaud, A. Galindo and G. Jackson, Ind. Eng. Chem. Res., 2004, 43, 6871-6889.

40 A. van Pelt, C. J. Peters, J. de Swaan Arons and U. K. Deiters, J. Chem. Phys., 1995, 102, 3361.
41 J. Lachwa, J. Szydlowski, V. Najdanovic-Visak, L. P. Rebelo, K. R. Seddon, M. N. Da Ponte, J. M. Esperança and H. J. Guedes, J. Am. Chem. Soc., 2005, 127, 6542-6543.

42 D. G. Green and G. Jackson, J. Chem. Phys., 1992, 97, 8672-8691.

43 C. McCabe, A. Gil-Villegas and G. Jackson, J. Phys. Chem. B, 1998, 102, 4183-4188.

44 A. Galindo and F. J. Blas, J. Phys. Chem. B, 2002, 106, 4503-4515.

45 A. Valtz, A. Chapoy, C. Coquelet, P. Paricaud and D. Richon, Fluid Phase Equilib., 2004, 226, 333-344.

46 M. C. dos Ramos, F. J. Blas and A. Galindo, Fluid Phase Equilib., 2007, 261, 359-365.

47 R. Koningsveld, Br. Polym. J., 1975, 7, 435-466.

48 D. McIntyre, N. Rounds and E. Campos-Lopez, Polym. Prepr., 1969, 10, 531-537.

49 R. Koningsveld, L. A. Kleintjens and H. M. Schoffeleers, Pure Appl. Chem., 1974, 39, 1-32.

50 L. P. Rebelo and W. A. Van Hook, J. Polym. Sci., Part B: Polym. Phys., 1993, 31, 895-897.

51 H. Tanaka, AIP Conf. Proc., 1992, 238-239.

52 H. Schafer-Soenen, R. Moerkerke, H. Berghmans, R. Koningsveld, K. Dusek and K. Solc, Macromolecules, 1997, 30, 410-416.

53 E. Nies, A. Ramzi, H. Berghmans, T. Li, R. K. Heenan and S. M. King, Macromolecules, 2005, 38, 915-924.

54 K. Van Durme, G. Van Assche, E. Nies and B. Van Mele, J. Phys. Chem. B, 2007, 111, 1288-1295.

55 S. Vanhee, R. Koningsveld, H. Berghmans and K. Šolc, J. Polym. Sci., Part B: Polym. Phys., 1994, 32, 2307-2309.

56 E. Loozen, K. Van Durme, E. Nies, B. Van Mele and H. Berghmans, Polymer, 2006, 47, 7034-7042.

57 F. E. Pereira, G. Jackson, A. Galindo and C. S. Adjiman, Comput. Chem. Eng., 2012, 36, 99-118.

58 W. Wagner, A. Saul and A. Pruss, J. Phys. Chem. Ref. Data, 1994, 23, 515-527.

59 G. Jackson, Mol. Phys., 1991, 72, 1365-1385.

60 M. S. Wertheim, J. Stat. Phys., 1984, 35, 19-34.

61 K. Heremans and L. Smeller, Biochim. Biophys. Acta, Protein Struct. Mol. Enzymol., 1998, 1386, 353-370.

62 L. Smeller, Biochim. Biophys. Acta, Protein Struct. Mol. Enzymol., 2002, 1595, 11-29.

63 R. Winter, Biochim. Biophys. Acta, Protein Struct. Mol. Enzymol., 2002, 1595, 160-184.

64 R. Milo, BioEssays, 2013, 35, 1050-1055.

65 P. Masson and C. Clery, High Pressure Bioscience and Biotechnology, 1996, pp. 117-126.

66 V. Lullien-Pellerin and C. Balny, Innovative Food Sci. Emerging Technol., 2002, 3, 209-221.

67 M. Gross and R. Jaenicke, Eur. J. Biochem., 1994, 221, 617-630.

68 K. Takai, K. Nakamura, T. Toki, U. Tsunogai, M. Miyazaki, J. Miyazaki, H. Hirayama, S. Nakagawa, T. Nunoura and K. Horikoshi, Proc. Natl. Acad. Sci. U. S. A., 2008, 105, 10949-10954.

69 A. Zipp and W. Kauzmann, Biochemistry, 1973, 12, 4217-4228. 